\title{
Frictional Properties of the Ultra-thin Films Constructed with Fluorocarbon Rods
}

\author{
Kenji Hisada*, Akiko Hino, Saori Inoue, and Teruo Hori \\ Fiber Amenity Engineering Course, Graduate School of Engineering, University of Fukui, 3-9-1 Bunkyo, Fukui 910-8507, Japan
} Fax:+81-776-27-8747, e-mail: hisada@acbio2.acbio.fukui-u.ac.jp

\begin{abstract}
We have investigated the frictional properties of the Langmuir-Blodgett (LB) films by lateral force microscope. The LB films were prepared with the amphiphiles having fluorocarbon or hydrocarbon tails. The frictional behavior was discussed as a function of the terminal atom of hydrophobic end and the size of the hydrophilic groups. The films prepared with F-terminated amphiphiles showed friction force lower than those prepared with corresponding $\mathrm{H}$-terminated amphiphiles. The monolayers of the fluorinated diols gave higher friction at lower normal load. When the normal load was less than $10 \mathrm{nN}$, large hydrophilic head of diol increases the free volume around the hydrophobic chain. The large free volume accelerates energy dissipation within the monolayer, which enlarges the friction at lower load.
\end{abstract}

Key words: Langmuir-Blodgett film, Fluorocarbon rod, Energy dissipation, Tilted configuration, Lateral force microscopy

\section{INTRODUCTION}

As a result of the research on ultrathin organic films over past decades, it is now possible to prepare monolayers with known microscopic and macroscopic organization [1]. Monolayers produced at the air/water interface can be transferred to a solid surface by the Langmuir-Blodgett (LB) technique. Monolayers can be prepared from solutions as well by self-assembly. The well-ordered systems has led to their use in investigations of a number physical phenomena at the surface, which is called as tribology. Although the phenomenological study on friction has a long history at the macroscopic scale [2-4], frictional processes was hard to study at the atomic scale. With the well-characterized surfaces, it has become possible to correlate mechanical properties (adhesion, friction and wear) with the molecular structure of the sliding interfaces.

A key element in the tribological studies on monolayers is the use of scanning force microscopy (SFM), which can visualize in situ electrically insulating films with high resolution [5-10]. SFM observations revealed the topology of a surface and, they provided information about the friction as well when operated in lateral force mode [11]. Such studies examined the effects of chain length [12-14] and terminal group [15] on nanoscopic friction. One of unique results obtained by SFM measurement was that the monolayers constructed with fluorocarbon rods have higher friction than those having hydrocarbon tails $[16,17]$. As discussed by McFarlance and Tabor, high friction force is associated with a large adhesion between the surfaces in a macroscopic friction [18]. However, in the nanoscopic measurement, lower adhesive fluorocarbon domains gave higher friction. Salmeron investigated a mechanism of nanoscopic friction by using lateral force microscope (LFM) and sum-frequency generation, and he concluded that the energy dissipation contribute the friction observed with LFM [19]. Krim also reported the nanoscopic friction is associated with the energy dissipation through acoustic oscillation on the metal surfaces $[20,21]$.

We report here the frictional force measured with LFM for Langmuir-Blodgett films prepared with amphiphiles having fluorocarbon rod or alkyl chain. The nanoscopic friction was discussed taking into account the adhesion and the plastic deformation of hydrophilic domains.

\section{EXPERIMENTAL}

\subsection{Materials}

1-O-palmityl-rac-glycerol (ETD16h) was purchased from Sigma Chemical Co. Perfluorononanoic acid (FCA9f) and 9H-hexadecafluorononanoic acid (FCA9h) were purchased from Daikin Chemicals Sales Co., Ltd. $(4,4,5,5,6,6,7,7,8,8,9,9,10,10,11,11,11$-heptadecafluoro)1,2-undecanediol (FD8f) and 1-O-(2,2,3,3,4,4,5,5,6,6,7, 7,8,8,9,9-hexadecafluorononyl)-rac-glycerol (FTED9h) were synthesized from 3-perfluorooctyl-1,2-epoxy propane (Daikin Chemicals Sales Co., Ltd.) and glycidyl 2,2,3,3,4,4,5,5,6,6,7,7,8,8,9,9-hexadecafluorononyl ether (Aldrich Chemical Co.), respectively. Trifluoroacetic acid was used to open the oxirane ring, and the adduct was hydrolyzed with aqueous solution of sodium carbonate to prepare diol derivatives. The products were isolated through column chromatography on silica gel and were identified by ${ }^{1} \mathrm{H}-\mathrm{NMR}$ spectroscopy. The structures and abbreviations for amphiphiles are shown in Fig.1. It should be noted here that the diols, ETD16h, FD8f and FETD9h, have hydrophilic head relatively larger than the carboxylic acids, FCA9f and FCA9f.

\subsection{Surface pressure-area isotherm}

Surface pressure $(\pi)$ - area $(A)$ isotherms were measured with a Langmuir trough (HBM AP3, Kyowa Kaimenkagaku Co. Ltd.). The dilute solutions of amphiphiles were spread onto water purified with EasyPure RF (Barnstead) $(\rho>18$ 


$$
\begin{array}{ll}
\mathrm{OH} & \begin{array}{l}
\mathrm{R}: \mathrm{H}\left(\mathrm{CH}_{2}\right)_{15} \mathrm{CH}_{2} \mathrm{O} \text { (ETD16h) } \\
\mathrm{R}: \mathrm{H}\left(\mathrm{CF}_{2}\right)_{8} \mathrm{CH}_{2} \mathrm{O} \text { (FETD9h) } \\
\mathrm{R}: \mathrm{F}\left(\mathrm{CF}_{2}\right)_{8}(\mathrm{FD} 8 \mathrm{f})
\end{array} \\
\mathrm{R}+\mathrm{CF} \frac{1}{2 / 8} \mathrm{C}_{\mathrm{OH}}^{\prime \prime} & \begin{array}{l}
\mathrm{R}: \mathrm{H} \text { (FCA9h) } \\
\mathrm{R}: \mathrm{F}(\mathrm{FCA} 9 \mathrm{f})
\end{array}
\end{array}
$$

Fig.1. Chemical structures of amphiphiles and their abbreviations.

$\mathrm{M} \Omega \mathrm{cm})$. ETD16h was dissolved in a mixed solvent of $n$-heptane and ethanol ( $9: 1$ by volume) and fluorinated amphiphiles were dissolved Asahiklin $^{\mathrm{TM}} \mathrm{AK}-225$ AES (a mixture of $\mathrm{CF}_{3} \mathrm{CF}_{2} \mathrm{CHCl}_{2}$ and $\mathrm{CClF}_{2} \mathrm{CF}_{2} \mathrm{CHClF}$, Asahi Glass Co., Ltd.). The film morphology was observed with homemade Brewster-angle microscope (BAM) at the air-water interface.

\subsection{Transfer of the assembled films}

The monolayers were transferred from water surface onto polished silicon wafers (Shin-Etsu Chemical Co., Ltd., DP050000) for lateral force microscopy. The wafers were sonicated with toluene, acetone, ethanol, and distilled water, successively. After then the substrate was cleaned by irradiating $\mathrm{O}_{2}$ plasma (Yamato Science Co. Ltd., PR300) to remove a trace of organic contaminants. The cleaning procedure made the silicon surface hydrophilic. The spread films were compressed at a speed of $15 \mathrm{~cm}^{2} / \mathrm{min}$. Monolayers of ETD16h, FD8f and FTED9h were transferred onto solid substrate by the Langmuir-Blodgett method. The deposited surface pressures were $15 \mathrm{mN} / \mathrm{m}$ for ETD16h and $20 \mathrm{mN} / \mathrm{m}$ for FD8f and FETD9h. The transfer ratios of these monolayers were more than 0.85 . The monolayers of FCA9f and FCA9h were deposited by the inverse Langmuir-Scheafer method [22,23]. Monolayers having fluorocarbon rods were deposited at a molecular area $0.30 \mathrm{~nm}^{2} /$ molecule.

\subsection{Lateral Force Microscopy}

Nanoscopic friction of the deposited films were performed with a scanning force microscope (Seiko Instruments Inc., SPI3700). A normal spring constant of rectangular cantilevers was of $0.10 \mathrm{~N} / \mathrm{m}$. The cantilever had a pyramidal $\mathrm{Si}_{3} \mathrm{~N}_{4}$ tip and its radius of curvature was $20 \mathrm{~nm}$ (Olympus Optical Co., Ltd., Micro Cantilever OMCL- RC800PSA). Applied normal loads were $0.087-40 \mathrm{nN}$ during LFM measurement. A $150-\mu \mathrm{m}$ scanner table was used for all measurements. Friction forces were obtained by recording traces of cantilever torsional deformation (friction loops) over scan areas of $40 \mu \mathrm{m} \times 40 \mu \mathrm{m}$ with a sliding velocity of $80 \mu \mathrm{m} / \mathrm{s}$. Friction forces were averaged for more than 100 data points per sample at different locations on the monolayer surface. Representative friction loops are shown in the inserted of Fig.4. For each friction loop, the difference in the friction signal (voltage) between the forward and reverse scans was converted to friction force by applying Hooke's law. The torsional spring constant of the cantilever was calculated with the

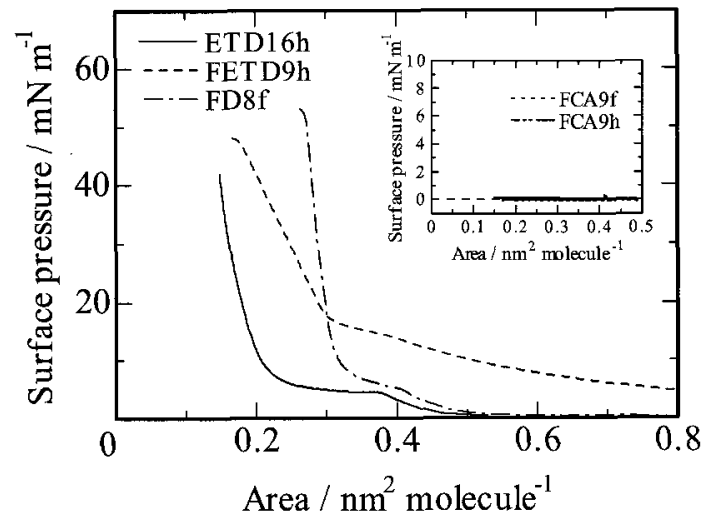

Fig.2. Surface pressure $(\pi)$ - area (A) isotherm for ETD16h ( $\longrightarrow$, FETD9h $(---)$, FD8f $(-\bullet-\bullet-)$ The inset shows $\pi$-A isotherm for FCA9f $(\cdot \cdots)$ and FCA9h $(-\cdots \cdots-\cdots)$.

geometry and Young's modulus of silicon nitride [24].

\section{RESULTS AND DISCUSSION}

3.1 Surface pressure - area isotherms

Figure 2 shows $\pi-A$ isotherms for monolayers on ultrapure water at $20{ }^{\circ} \mathrm{C}$. The $\pi-A$ isotherm for ETD16h shows obvious plateau region from 0.38 to 0.24 $\mathrm{nm}^{2} /$ molecule. At this plateau region, liquid expand (LE) phase coexisted with liquid condensed (LC) phase and the proportion of LC phase increased with compressing the monolayer [25]. After the LE-LC phase transition, the isotherm showed steep rise resulting in the closely packed monolayer. The monolayers of fluorinated diols, FETD9h and FD8f, also showed LE-LC phase transition following steep rise. The cross-sectional area for fluorocarbon rod $(0.37$ $\mathrm{nm}^{2} /$ chain) is larger than that for alkyl chain $(0.19$ $\mathrm{nm}^{2} /$ chain) [26], which causes that the steep rise in $\pi-A$ isotherm appeared at the larger molecular area for the fluorinated diols. The plateau regions for the fluorinated diols were narrower than that for ETD16h and the profile resembled that of ETD16h and 1-palmytoyl-rac-glycerol at elevated temperatures $[27,28]$. The activation of thermal motion looses the molecular packing at elevated temperature, which makes unstable the LC phase. The fluorocarbon rod has lower cohesive forces than the alkyl tails because of its quite nonpolar feature. The feature also makes LC phase unstable, so the phase transition takes place at the molecular area close to its intrinsic molecular area. The monolayers of FCA9h and FCA9f collapsed at quite low surface pressure, so the monolayers were deposited at the molecular area of $0.35 \mathrm{~nm}^{2} /$ molecule.

\subsection{Brewster-angle microscopy}

The monolayer of ETD16h gave similar BAM image with a monolayer of 1-monopalmytoyl-rac-glycerol [29]. There are cardioid-shaped domain containing wedge-shaped segments; which show that alkyl tail aligned with same molecular tilt azimuth in a segment in a micrometer scale (Fig.3(a)). On the other hand, no condensed domain was recognized in the BAM images for the monolayers prepared with the diols having fluorocarbon rods (Fig.3(b) and Fig.3(c)) while their 
(a) ETD16h

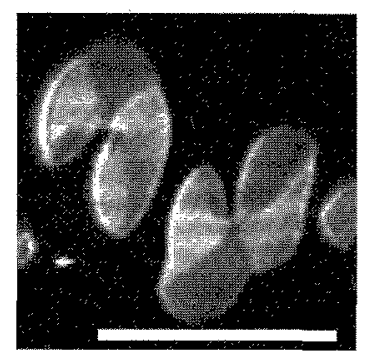

(b) FETD9h

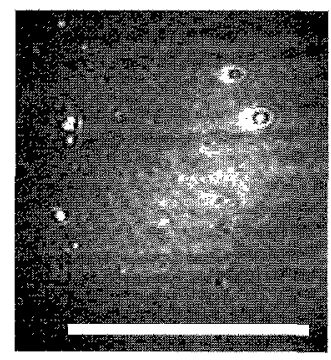

(d) FCA9h

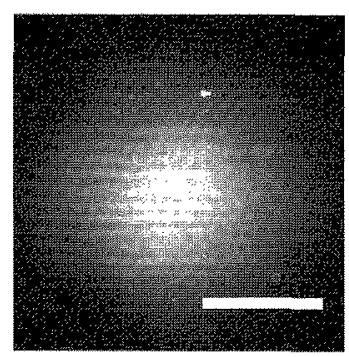

(e) FCA9f

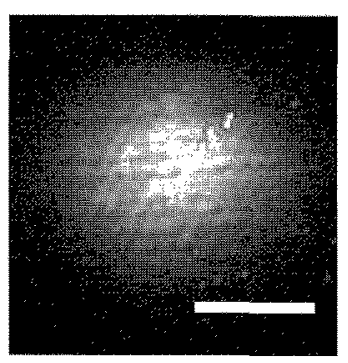

Fig.3. Brewster angle microscopic images for the monolayers on the ultrapure water: (a) ETD16h, (b) FETD9h, (c) FD8f, (d) FCA9h, and (e) FCA9f. The white bar denotes $500 \mu \mathrm{m}$.

$\pi-A$ isotherms showed the LE-LC transition (Fig.2). These results suggest that the LC domains of fluorinated diols were smaller than the resolution of optical microscope, which was arising from low cohesive tendency of fluorocarbon rods. On the ultrapure water, FCA9h and FCA9f were observed as LE phase. The films were estimated as monolayer from the brightness of BAM images.

\subsection{Lateral force microscopy}

Figure 4 shows frictional force measured with LFM as a function of the loading force. F-terminated amphiphiles, FCA9f and FD8f, showed friction force lower than those for corresponding H-terminated amphiphiles, FCA9h and FETD9h. The results were consistent with the macroscopic friction, that is, high friction force is associated with a large adhesion between the surfaces [18]. However, unusual feature was observed for the monolayer prepared with diols having fluorocarbon rods, FETD9h and FD8f. These monolayers showed negative friction coefficients at lower load as shown in Fig.4. The negative frictional coefficient was obtained only when the amphiphiles

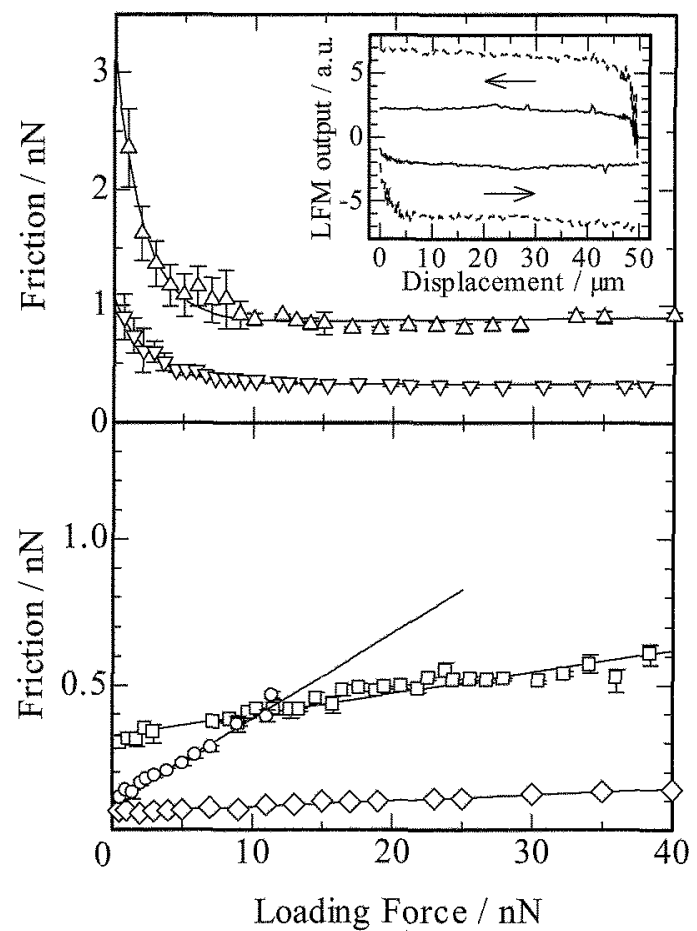

Fig.4. Frictional force as a function of the loading force for the monolayers deposited on Si wafer. $\triangle$ : FETD9h, $\nabla$ : FD8f, O: ETD16h, $\square$ : FCA9h, and $\diamond$ : FCA9f, Representative friction loops for FD8f monolayer are inserted in the graph. Applied loads were $0.7 \mathrm{nN}(--)$ and $30.7 \mathrm{nN}(-)$, respectively.

having relatively large hydrophilic head and fluorocarbon tails. When the hydrophilic heads were smaller, FCA9h and FCA9f, or the hydrophobic tail was alkyl chain, ETD16h, the friction coefficients were positive over the whole applied load range. LFM images were also observed simultaneously with the force measurements. All LB films were confirmed that they were not swept away by the LFM probe at these normal loads and therefore frictional force is mainly caused by a probe-film friction.

It was reported that the energy dissipation process is a key process in the microscopic friction obtained by the lateral force microscope [19-21]. We had reported that the friction force for polymer LB film had a maximum at specific shear rate [30]. The phenomenon was similar to the loss modulus for the viscoelastic materials. The loss modulus is closely related with energy dissipation in the materials. Action is inevitably followed by reaction. When the monolayer dissipate energy through physical phenomenon, it is necessary to add extra energy on the probe tip for continuous movement. In other words, friction becomes larger in such a case. The monolayers of FD8f or FETD9h had large free volume around the fluorocarbon rods because large hydrophilic head disturbed the aggregation of hydrophobic tails. In such a case, the monolayer deformed plastically with the large energy dissipation, and these monolayers showed larger friction coefficients at low normal load. Compression with higher load makes the film condense, and the monolayer became to deform elastically, storing the deformation energy, as shown in Fig.5(b). When 
(a)

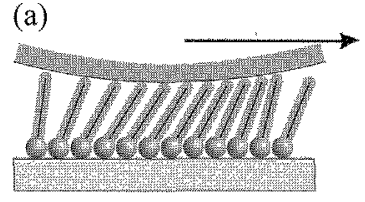

(c)

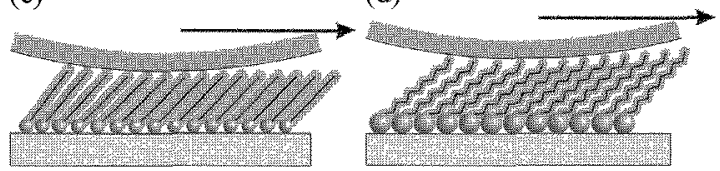

Fig.5. Schematic illustration of LB films during the shear deformation. The monolayer prepared with fluorocarbon rods having large heads at $(a, b)$, fluorocarbon rods having small heads (c), and alkyl tail having large heads (d). (a) shows the monolayer compressed at low load and (b) expresses the one compressed at high load.

fluorocarbon tail links with small hydrophilic head (Fig.5(c)) or amphiphile had alkyl tail (Fig.5(d)), the monolayer is in a condensed state even if the film is not compressed. Barrena et al. showed that the more than $35 \mathrm{nN}$ external load could induce chain tilting from the first tilted configuration to the second one [31]. So, the dense phases of hydrophobic tails would not change their tilted configuration at applying normal load, and the friction for these films should increase linearly with a frictional coefficient. In a microscopic driving device, lubricant should be atomically flat and the normal load will be quite low. Present results showed that the molecular packing in the lubricant layer and their deformation have strong influence in the frictional behavior.

\section{CONCLUSIONS}

We have investigated the frictional properties of the LB films with LFM. The LB films were prepared with the amphiphiles having alkyl or fluorocarbon tails. The frictional behavior was discussed as a function of the terminal atom in the hydrophobic tail and the size of the hydrophilic groups. F-terminated amphiphiles, showed friction force lower than those for corresponding $\mathrm{H}$-terminated amphiphiles. The monolayers of the fluorinated diols gave higher friction at lower normal load unusually. The larger friction at low load seems to be related to the plastic deformation of fluorocarbon diol monolayer having large free volume around fluorocarbon rods.

\section{ACKNOWLEDGEMENTS}

Authors want to show great thanks to Professor Yuji Tokunaga who gave us some information about the synthesis of FD8f and FETD9h. This work was supported by a Grant-in-Aid for Young Scientists (B) (No. 15750101) from the Ministry of Education, Culture, Sports, Science and Technology of Japan.

\section{REFERENCES}

[1] A. Ulman, "An Introduction to Ultrathin Organic Films: From Langmuir-Blodgett to Self-Assembly", Academic Press, Boston (1991).

[2] F.P. Bowden and D. Tabor, "Friction and
Lubrication of Solids", Oxford University Press, Oxford (1986).

[3] "Handbook of Micro/Nanotribology", Ed. By B. Bhushan, CRC Press, Boca Raton (1995).

[4] B.N.J. Persson, "Sliding Friction - Physical Principles and Applications", Springer, New York (1998).

[5] U. Srinivasan, M.R. Houston, R.T. Howe, and R. Maboudian, J. Microelectromech. Syst., 7, 252 (1998).

[6] Q. Dai, G. Vurens, M. Luna, and M. Salmeron, Langmuir, 13, 4401 (1997).

[7] C.P. Tripp, R.P.N. Veregin, M.N.V. McDougall, and D. Osmond, Langmuir, 11, 1858 (1995).

[8] M. Radmacher, R.W. Tillmann, M. Fritz, and H.E Gaub, Science, 257, 1900 (1992).

[9] R.M. Overney, E. Meyer, J. Frommer, D. Brodbeck, R. Lüthi, L. Howald, H.-J. Güntherodt, M. Fujihira, H. Takano, and Y. Gotoh, Nature, 359, 133 (1992):

[10] E. Meyer, R. Overney, D. Brodbeck, L. Howald, R. Lüthi, J. Frommer, and H.-J. Güntherodt, Phys. Rev. Lett., 69, 1777 (1992).

[11] R.W. Carpick and M. Salmeron, Chem. Rev., 97, 1163 (1997).

[12] X. Xiao, J. Hu, D.H. Charych, and M. Salmeron, Langmuir, 12, 235 (1996).

[13] M.T. McDermott, J.-B. Green, and M.D. Porter, Langmuir, 13, 2504 (1997).

[14] A. Lio, D.H. Charych, and M. Salmeron, J. Phys. Chem. B, 101, 3800 (1997).

[15] C.D. Frisbie, L.F. Rozsnyai, A. Noy, M.S. Wrighton, and C.M. Lieber, Science, 265, 2071 (1994).

[16] J. Fang and C.M. Knobler, Langmuir, 12, 1368 (1996).

[17] K. Kojio, A. Takahara, and T. Kajiyama, Langmuir, 16, 9314 (2000).

[18] F.P. Bowden and D. Tabor, "Adhesion between solid surfaces: the influence of liquid films, in Friction and Lubrication of Solids", Oxford University Press, Oxford, (1986) pp. 312-314.

[19] M. Salmeron, Tribol. Lett., 10, 69 (2001).

[20] J. Krim, Langmuir, 52, 4564 (1996):

[21] J. Krim, Lubr. Eng., 53, 8 (1997).

[22] K. Hisada and C.M. Knobler, Langmuir, 16, 9390 (2000):

[23] U. Gehlert, J. Fang, and C.M. Knobler, J. Phys. Chem. B, 102, 2614 (1998).

[24] G. Meyer and N.M. Amer, Appl. Phys. Lett., 57, 2089 (1990).

[25] U. Gehlert and D. Vollhardt, Langmuir, 18, 688 (2002)

[26] C.W. Bunn and E.R. Howells, Nature, 174, 549 (1954).

[27] U. Gehlert, G. Weidemann and D. Vollhardt, $J$. Colloid Interface Sci., 174, 392 (1995):

[28] N. Nandi and D. Vollhardt, Chem. Rev., 103, 4033 (2003).

[29] D. Vollhardt, U. Gehlert, and S. Siegel, Colloids Surf. A, 76, 187 (1993).

[30] K. Hisada and T. Hori, Koubunshi Kakou, 53, 37 (2004).

[31] E. Barrena, S. Kopta, D.F. Ogletree, D.H. Charych, and M. Salmeron, Phys. Rev. Lett., 82, 2880 (1999). 